\title{
L'Acheronte dantesco: morte del Pellegrino e della poesia
}

0 . All'inizio del viaggio infernale, poco dopo aver varcato la porta dell'Inferno (Inf. 3.1-21) e osservato gli ignavi (3.35-36), Dante arriva al fiume Acheronte. Lungo la sponda del "fiume della morte," dopo l'incontro con Caronte e la spiegazione di Virgilio circa la condizione dei dannati, si verifica un terremoto che causa lo svenimento di Dante:

Finito questo, la buia campagna tremò sì forte, che de lo spavento la mente di sudore ancor mi bagna.

La terra lagrimosa diede vento, che balenò una luce vermiglia la qual mi vinse ciascun sentimento; e caddi come l'uom cui sonno piglia.

A questo punto né Dante Pellegrino si rende conto del significato del terremoto e dello svenimento né Dante Poeta offre al lettore alcuna spia per interpretare il testo. Poiché il poeta non spiega ora questi avvenimenti, al lettore non resta altro che continuare la lettura fino al momento in cui sarà possibile, partendo dal testo dantesco, offrire una spiegazione sia del terremoto che dello svenimento. Sappiamo tuttavia che è il terremoto, assieme con il vento e la luce vermiglia, a causare la perdita dei sensi del Pellegrino. La sua "caduta" (si noti il verbo usato dal poeta per descrivere le conseguenze dello svenimento) è uguagliata a quella dell'uomo che è vinto dal sonno: similitudine strana, questa, poiché non fa parte della nostra esperienza vedere un uomo cadere quand'è sopraffatto dal sonno; e quindi osservazione fondamentale per la valutazione del significato, per ora recondito, del terremoto e dello svenimento. È chiaro dunque che il fenomeno tellurico che scuote tutto il luogo infernale non può in definitiva essere preso in esame separatamente dallo svenimento, che tuttavia colpisce solo Dante Pellegrino. In realtà forse è possibile 
speculare fin d'ora che la perdita dei sensi di Dante costituisca lo scopo fondamentale dello straordinario fenomeno tellurico.

La prima indicazione esplicita di un altro terremoto ci è data dalla bocca di Malacoda, nella quinta bolgia del cerchio ottavo (21.10614). Vero è che Malacoda, pur non parlando di alcun terremoto in particolare, non solo descrive gli effetti rovinosi, causati da un gran terremoto, sul regno di Satana, ma ne offre anche le coordinate temporali per individuarne l'origine. Veniamo così a sapere che $\mathrm{i}$ ponti rotti, e presumibilmente anche le altre "ruine" che Dante aveva notato durante la sua discesa infernale, sono da rapportare alla crocifissione di Cristo e al fenomeno tellurico che l'accompagnò.

1. Il narrato biblico della morte di Cristo nel vangelo di Matteo ci offre gli elementi necessari per la spiegazione del terremoto infernale:

Et ecce velum templi scissum est in duas partes a summo usque deorsum: et terra mota est, et petrae scissae sunt, et monumenta aperta sunt: et multa corpora sanctorum, qui dormierant, surrexerunt. (Matt. 27.51-52)

Il narrato biblico e il testo dantesco giustificano ampiamente la proposta che il terremoto che accompagna la morte di Cristo sia lo stesso che causa le "ruine" nel regno di Satana. Infatti, alla morte di Cristo tiene dietro la sua discesa agli inferi: avvenimento, questo, che Virgilio stesso descrive a Dante, in seguito alla sua domanda, in termini precisi e rivelatori (4.52-63). Cristo, il "possente, / con segno di vittoria coronato," si assoggetta non al peccato e a Satana ma alle conseguenze del peccato istigato da Satana stesso, cioè la morte; a questa sottomissione temporanea tiene dietro la vittoria di Cristo su Satana e sul suo regno. Di qui la "ruina" dei ponti e di altri luoghi infernali (5.28-36 e 12.4-10, secondo Charles S. Singleton 475) causati del terremoto, e la liberazione delle anime giuste dal Limbo tramite la discesa di Cristo agli inferi. Singleton scrive:

E se ora dalla fitta rete di tutti questi segni ci voltiamo a guardare la parola tomba, che compare verso la fine della salita per uscire dall'Inferno, essa assumerà per noi uno speciale significato. Lì indicava senza dubbio la tomba di Satana; ma quando il pellegrino esce da essa proprio prima dell'alba della Domenica di Pasqua, sarà difficile che il lettore cristiano non trovi in tale fatto una ricchezza di significato. $(487-88)^{1}$

Senonché Singleton, nonostante gli stretti rapporti che egli sviluppa 
fra la "discesa" di Cristo, subito dopo la morte in croce, e la "discesa" di Dante Pellegrino, non sembra mettere in luce ulteriori rapporti fra l'inizio di questi due viaggi infernali. Ciò che ci interessa sottolineare a questo punto è che l'inizio del viaggio dantesco, che segue di poco l'ora della morte di Cristo in croce, la sesta ora del Venerdi Santo (21.112-14), è appunto contrassegnato da un terremoto e dalla perdita dei sensi del Pellegrino: il fenomeno tellurico che scuote "la buia campagna" deve necessariamente rapportarsi al terremoto che accompagna la morte di Cristo in croce, alla quale occorre anche riferire la perdita dei sensi di Dante; infine, la discesa infernale di Dante Pellegrino che dalla sera del Venerdi Santo alla mattina della Domenica di Risurrezione è racchiuso nelle viscere della terra trova il suo modello mitico e la sua giustificazione soteriologica nel Christuserlebnis.

2. Prima di procedere all'analisi dei rapporti fra il descensus ad inferos di Cristo, preceduto dalla sua morte reale, e il descensus ad inferos di Dante, preceduto dal suo svenimento o morte simbolica, occorre considerare il terremoto dantesco nei suoi rapporti con il terremoto che nell'Eneide-testo fondamentale nella genesi della Commedia - caratterizza l'ingresso della Sibilla e di Enea nell'Ade:

Ecce autem primi sub lumina solis et ortus sub pedibus mugire solum et iuga coepta moveri silvarum, visaeque canes ululare per umbram, adventante dea.

(En. 6.255-58)

La formula che introduce il fenomeno- "ecce autem"-formula che ha un preciso corrispondente nel dantesco "Ed ecco" (3.82), è spesso usata per contraddistinguere avvenimenti improvvisi che determinano lo sviluppo d'un'azione e che causano paura o ammirazione (Aeneidos comm. ad loc.). In realtà tre elementi connotano il terremoto virgiliano: 1. il "muggire" del suolo sotto i piedi; 2. lo scuotersi delle vette degli alberi della foresta; 3 . il latrare dei cani. Il primo e il terzo elemento sono convenzionali nella letteratura classica antica. ${ }^{2}$ Il secondo e il terzo elemento virgiliano non caratterizzano l'ingresso infernale della Commedia, in cui il terremoto non viene descritto metaforicamente come in Virgilio ("mugire solum") bensì in termini specifici e realistici ("la buia campagna / tremò sì forte") con l'ag- 
giunta di due elementi, estranei al testo dell'Eneide, e cioè il "vento" e "una luce vermiglia," che, secondo la meteorologia dantesca, sono costitutivi del fenomeno tellurico. In realtà anche qui è valido un principio interpretativo che a noi sembra fondamentale, pur nella sua evidente immediatezza: se è vero che, da un lato, le somiglianze testuali suggeriscono un contesto interpretativo generale (nella fattispecie, il terremoto virgiliano), dall'altro sono le disuguaglianze ad offrire la chiave d'interpretazione vera e propria.

Il terremoto, dunque, elemento comune in entrambi i testi, viene descritto in termini sostanzialmente diversi. Mentre in ambedue $i$ casi il terremoto è da porsi in relazione con l'ingresso nell'aldilà, nell'Eneide l'eroe e la Sibilla non sono ancora entrati nella caverna infernale; al contrario, nella Commedia i due pellegrini sono già arrivati alla riva del fiume Acheronte. Inoltre il testo virgiliano suggerisce un rapporto, presumibilmente di dipendenza, fra il terremoto e la presenza della divinità ("adventante dea"); la Commedia non offre immediatamente un tale rapporto, ma è l'analisi di altri episodi danteschi in cui è presente il fenomeno tellurico a proporci l'episodio come una specifica manifestazione della divinità in un momento cruciale del viaggio di Dante Pellegrino (si veda Inf. 9.64-66).

Del tutto discriminante, per quanto concerne i due fenomeni tellurici, è la differenza nell'atteggiamento di Enea e di Dante di fronte al terremoto. Nell'Eneide al fenomeno tellurico tengono dietro il grido imperioso della Sibilla, diretto ai "profani," di allontanarsi; l'invito, rivolto ad Enea, di avanzare con la spada sguainata e con coraggio; e l'ingresso della Sibilla e dell'eroe nell'antro (6.258-63). Sebbene la Sibilla sproni l'eroe ad aver coraggio, in realtà l'eroe non sembra aver bisogno di alcuna esortazione, come esprime lucidamente 6.263 ("ille ducem haud timidis vadentem passibus aequat"). In questo contesto il testo della Commedia si stacca decisamente dal modello classico: il timore e la paura che si sono alternati nell'animo del Pellegrino fin dall'inizio del viaggio (1.6-7, 15, 19, ecc.), raggiungono in questa congiuntura un apice che Dante non oltrepasserà nemmeno quando si troverà davanti al "gran vermo," la più terrificante delle visioni infernali (34.22-27). Infatti il terremoto, il vento e la luce vermiglia vincono "ciascun sentimento" del Pellegrino che tramortisce. Quest'esperienza è così terrificante—nota lo scrittore-che il solo ricordo gli irriga ancora di sudore la fronte. In breve: l'at- 
teggiamento di Enea di fronte al terremoto, al momento d'entrare nell'antro, mette in evidenza la "sufficienza" dell'eroe che intraprende il viaggio nell'ald̛ilà, mentre al contrario l'atteggiamento di Dante è indice della sua "insufficienza."

La verifica di questa differenza fondamentale fra il viaggio nell'oltretomba di Dante Pellegrino e quello di Enea-viaggio di un cristiano, il primo; di un pagano, il secondo-è riscontrabile nel "segno" che permette all'uno e all'altro l'ingresso nell'aldilà. Nel poema virgiliano la Sibilla mostra a Caronte il ramus aureus (6.403-10) che Enea aveva raccolto nel bosco seguendo le istruzioni della maga. Nel poema dantesco, Virgilio, la guida assegnata al Pellegrino dalle tre donne sante, profferisce a Caronte non un dono materiale, per quanto pieno di valori simbolici, come nell'Eneide (si veda il commento serviano), bensì impone al nocchiere infernale un comando divino (3.94-96).

Di qui possiamo inferire ulteriori differenze fra i due transitus dell'Acheronte. Al profferire del ramo d'oro il Caronte virgiliano si calma; alle parole di Virgilio il Caronte dantesco tace ma ovviamente non s'acquieta. La diversità nella reazione dei due Caronti (e di qui diventa palese che si tratta proprio di due personaggi diversi) si manifesta anche nella trasformazione d'animo che si attua nel Caronte virgiliano. Questi non solo si rappacifica ("tumida ex ira tum corda residunt," 6.407), ma, per così dire, passa dalla parte di Enea, di cui ora ammira il dono venerabile, quella verga fatale che non vedeva più da lungo tempo (6.408-9). E quindi accoglie subito Enea nella barca. Il testo virgiliano sembra mettere ulteriormente in luce l'ammirazione di Caronte per Enea il quale, entrato nella barca, sovrasta gli astanti con la sua forma immane:

inde alias animas, quae per iuga longa sedebant,
deturbat laxatque foros; simul accipit alveo
ingentem Aeneam. gemuit sub pondere cumba
sutilis et multam accepit rimosa paludem.
tandem trans fluvium incolumis vatemque virumque
informi limo glaucaque exponit in ulva.

Precisamente a motivo del vencrabile donum o virga fatalis, non v'è più opposizione fra Enea ("corpus vivum") e Caronte, il quale accoglie l'eroe nella barca. Enea sembra così assumere una statura simile 
o addirittura superiore ("ingentem Aeneam") a quella di Caronte, il quale infine depone incolumi ("incolumis"), al di là del fiume, la veggente e l'eroe.

Fondamentali sono quindi gli elementi che distinguono l'ingresso di Enea nell'aldilà dall'ingresso di Dante nell'Inferno: tramite il dono del ramo d'oro Caronte non solo viene messo a tacere ma sviluppa anche ammirazione per l'eroe; a parte il terremoto che accompagna l'appressarsi della dea all'antro infernale, nessun avvenimento straordinario caratterizza il passaggio dell'Acheronte virgiliano; Enea non teme né perde la conoscenza, anzi il suo passaggio dal regno dei vivi a quello dei morti è appunto contraddistinto da un continuo stato di coscienza e da una manifestazione di superiorità verso gli astanti o le circostanze esteriori, come è palese dalla descrizione di Enea, ritto sulla barca, durante il tragitto e dal suo arrivo, incolume, sull'altra sponda.

Contrariamente ad Enea, il Pellegrino perde la conoscenza e tramortisce, esperisce quindi nella propria persona i segni della morte. ${ }^{3}$ Nell'Eneide, da un lato, il valore discriminante assoluto che permette il passaggio è la contrapposizione fra corpora viva e corpora morta, che la necessità della sepoltura mette ancor più in rilievo. Nella Commedia, dall'altro, la sepoltura dei corpi non ha più nessun valore determinante nella scelta di Caronte, che accoglie nella barca tutti coloro che "cadono" lungo la riva dell'Acheronte. Per Caronte ciò che determina il passaggio dell'Acheronte, passaggio non invocato come nell'Eneide ("stabant orantes primi transmittere cursum / tendebantque manus ripae ulterioris amore," 6.314-5) ma temuto e odiato, è la condizione stessa dell'anima: prava e morta nell'ira di Dio.

Nel contesto del passaggio dell'Acheronte, la contiguità tematica del testo dantesco e virgiliano rivela a prima vista una comunanza di elementi contenutistici e di motivi poetici. In realtà le differenze, ovvie, implicite o recondite, sono tali e di così gran valore da mettere in luce un fatto macroscopico, rimasto nascosto solo a chi nella Commedia è in cerca di elementi formali a parte dei suoi significati spirituali: l'auctor vuole infatti segnalare, tramite l'imitazione contrapposta a una differenziazione sistematica, che il viaggio dantesco si attualizza per cause e scopi completamente diversi da quelli del viaggio di Enea. Quindi la differenza fra il primo e il secon- 
do è fondamentale, e le molteplici spie del testo dantesco rivelano il concetto fondamentale alla base di questa sostanziale differenza: Dante Pellegrino sta per intraprendere il viaggio nell'aldilà in virtù d'un privilegio la cui origine s'affonda nel mistero di Cristo il cui descensus/ascensus Dante s'accinge ora ad imitare.

3. Nelta Bibbia il terremoto e gli altri avvenimenti che accompagnano la morte di Cristo non possono essere visti che come una manifestazione della divinità. Tremori, tuoni, folgori, oscuramenti caratterizzano in genere il giorno del Signore, le teofanie, come la manifestazione divina a Mosè sul monte Sinai, e i due avvenimenti escatologici per eccellenza, ambedue visti in diretta dipendenza con la morte di Cristo: la distruzione di Gerusalemme e la fine del mondo.

Quali manifestazioni della divinità, cui sono sottomesse le forze della natura, nella Bibbia il terremoto, il vento e la folgore incutono timore nell'uomo (si veda la reazione del centurione ai piedi della croce); nella Commedia, come s'è visto, avvenimenti simili connotano la presa di possesso di Cristo sul regno di Satana e, di qui, altri eventi connessi con il viaggio dantesco o la salvezza delle anime purganti: la venuta del messaggero per aprire le porte della città di Dite (Inferno 9) e l'annuncio della purificazione delle anime purganti, come viene illustrato dalla liberazione di Stazio (Purgatorio 20). ${ }^{4}$

Nel terzo canto dell'Inferno, il terremoto che scuote la campagna sembra dover interpretarsi non solo quale teofania in genere bensì quale manifestazione della potenza divina che si rivelò alla morte di Cristo e alla presa di possesso del regno di Satana e che caratterizzerà anche la fine dei tempi, quando Cristo ritornerà per giudicare i vivi e i morti. Entro questo contesto, dunque, l'ingresso di Dante nell'oltretomba e nel regno di Satana, come tutto il viaggio dantesco, va interpretato come manifestazione diretta della volontà divina, secondo quanto le anime dannate e purganti si sentono proclamare più e più volte da Virgilio e da Dante. In quanto manifestazione della volontà divina, questa discesa nell'Inferno è configurata secondo l'archetipo del descensus di Cristo, il "possente / con segno di vittoria coronato," il primo fra i redenti, il Signore della vita e della morte. 
Il descensus di Dante, quindi, situato entro questo contesto escatologico, è quello dell'uomo peccatore che, per raggiungere la salvezza, deve imitare Cristo nella sua morte e nella sua discesa agli inferi. È quanto dice Beatrice stessa nel suo primo incontro con Dante in cima alla montagna del Purgatorio e in una fondamentale digressione teologica in Paradiso:

Tanto giù cadde, che tutti argomenti

a la salute sua eran già corti,

fuor che mostrarli le perdute genti.

Per questo visitai l'uscio d'i morti, e a colui che l'ha qua sù condotto, li preghi miei, piangendo, furon porti.

(Purg. 30.136-41)

La pena dunque che la croce porse s'a la natura assunta si misura, nulla già mai sì giustamente morse;

e così nulla fu di tanta ingiura, guardando alla persona che sofferse, in che era contratta tal natura.

Però d'un atto uscir cose diverse: ch'a Dio ed a' Giudei piacque una morte; per lei tremò la terra e 'l ciel s'aperse.

(Par. 7.40-48)

Quale momento essenziale della sua imitatio di Cristo, anche Dante, nel suo descensus nell'aldilà, non può non esperire la morte, la quale, come conseguenza della colpa originale, del peccato individuale e conditio sine qua non per l'ingresso nell'oltretomba, si attualizza nella perdita della conoscenza e nella caduta (paragonata al sonno) del Pellegrino.

Il nesso fondamentale che esiste fra il terremoto infernale e il terremoto narrato dall'evangelista Matteo, fra lo svenimento del Pellegrino e la morte di Cristo, determina il contesto entro cui vanno anche situati gli avvenimenti che tengono dietro al terremoto, allo svenimento di Dante e al passaggio dell'Acheronte.

Dopo la morte Cristo è sepolto, discende agli inferi, risorge e ascende quindi alla destra del Padre, da dove ritornerà alla fine dei tempi per giudicare i vivi e i morti. Cristo dunque non solo entra nell'aldilà ma inaugura i tempi messianici e annuncia anche la parusia. ${ }^{5}$ Dante che attraversa l'Acheronte e inizia un viaggio nel 
regno dei morti-trunsitus e viaggio caratterizzati da tipici segni apocalittici come terremoto, vento e folgore-lascia dunque dietro di sé l'aetum praesens ed entra in quello stato che è proprio dell'uomo dopo la morte. Osservazione ovvia, questa, ma fondamentale: la visione dantesca dell'Inferno, Purgatorio e Paradiso si svolge nell'aldilà, inteso e descritto come entità spaziale e temporale essenzialmente diversa dal mondo in cui viviamo. Questo è infatti l'argomento della Commedia, secondo quanto leggiamo nella lettera a Can Grande della Scala (12.2). Gli avvenimenti connessi con il passaggio dell'Acheronte caratterizzano l'ingresso di Dante Pellegrino nell'aldilà, inteso come entità spaziale e temporale e distinguono il narrato dei primi tre canti dal resto della Commedia. Il narrato pre-acheronteo-cioè lo smarrimento nella selva, l'incontro con Virgilio e la visione degli ignavi-fa ancora parte di questo mondo e di questo tempo; ${ }^{6}$ quanto avviene dopo il passaggio dell'Acheronte costituisce la visione dantesca. L'Acheronte quindi separa questo mondo dall'altro, il tempo presente dall'eternità, la realtà terrena dalla visione. ${ }^{7}$

4. Soffermiamoci su quest'ultima proposta-l'Acheronte separa la realtà dalla visione-al fine di confrontare i moduli narrativi della Commedia con quelli biblici. Il descensus ad inferos di Cristo realizza due valori fondamentali: il trionfo sulla morte e su Satana, che si verifica in termini assoluti e universali, e in quanto tale ha luogo alla morte e alla risurrezione di Cristo (Iannucci 51-81). Ma esso si verifica in termini individuali per ogni creatura che accetta Cristo e la morte con la speranza della risurrezione, accettazione che è la conditio sine qua non perchè la creatura possa risorgere. Dante Pellegrino deve quindi "morire" per poter ricevere la grazia della risurrezione. Lo svenimento fa sì che Dante diventi partecipe della mortalità che è comune a tutte le creature e pone quindi le condizioni necessarie per ricevere la grazia della risurrezzione.

L'analisi dei testi biblici che narrano la morte di Cristo mette in luce ulteriori rapporti con il testo dantesco. Dei quattro vangeli, quello di Matteo descrive il maggior numero di circostanze, incluso il terremoto, che accompagnano la morte di Cristo. Nel vangelo, per ordine, abbiamo i seguenti elementi: le tenebre, descritte con una precisa indicazione temporale (27.45); la morte di Cristo (27.46-50); 
i signa della morte, cioè lo spezzarsi del velo del tempio, il terremoto, l'apertura dei monumenti e la risurrezione di molti santi, il timore e l'affermazione di fede del centurione e degli astanti (27.51-54).Questi signa connotano la straordinarietà dell'evento e la presenza della divinità. Ciò che ci preme sottolineare ora è la sequenza temporale che li caratterizza. Abbiamo appunto, per ordine, prima le tenebre, poi la morte di Cristo, subito dopo il terremoto e gli altri segni che causano il timore degli astanti. Conclusione dell'episodio è la sepoltura di Cristo.

Il confronto con il testo dantesco rivela, per quanto concerne la cronologia degli avvenimenti, elementi simili e dissimili d'importanza fondamentali. Innanzitutto anche nella Commedia, come in Matteo, le tenebre avvolgono il luogo, come il testo sottolinea a più riprese sia all'inizio del canto ("l'aere sanza stelle," 3.23) come all'appressarsi all'Acheronte ("com'i' discerno per lo fioco lume," 3.75) e, ancor più esplicitamente, durante la sosta presso il fiume $(3.87,130 ; 4.10)$. Senonché, mentre le tenebre che scendono sul Golgota costituiscono un elemento straordinario-tutti e tre i sinottici concordano su questo fatto sottolineandone l'ora (Matt. 27.45; Marco 15.37; Luca 23.44)—nell'Inferno dantesco l'oscurità è presentata come caratteristica essenziale del "cieco mondo" (4.13), da rapportarsi quindi al peccato che è all'origine della creazione stessa dell'Inferno e della dannazione delle anime. Tuttavia è precisamente in questo rapporto essenziale fra tenebre e peccato che il testo evangelico e quello dantesco concordano, ovviamente entro un contesto simbolico o simbolico-teologico. Infatti, per quanto concerne le tenebre dei sinottici, il sole s'oscura per esprimere il dolore causato dalla morte del creatore ed anche per significare il peccato che è la causa della morte di Cristo. ${ }^{8}$

Il terremoto ha luogo dopo le parole di Virgilio (3.121-29), il quale spiega a Dante a) la condizione di quelli che muoiono nell'ira di Dio i quali discendono tutti all'Acheronte; b) il desiderio dei dannati di attraversare l'Acheronte, spronati dalla giustizia divina; c) e il motivo delle rimostranze di Caronte nei confronti di Dante. Il terremoto quindi va posto entro il contesto stabilito dalle parole di Virgilio, il quale qui annuncia la giustizia divina in atto nell'Inferno e poco dopo, nel canto seguente, descrive la discesa di Cristo nel Limbo quale "un possente, / con segno di vittoria coronato." 
Poiché il terremoto è segno della giustizia divina e manifestazione tcrribile della divinità, è possibile comprendere ciò che avviene al Pellegrino al suo verificarsi: egli infatti, spaventato e sopraffatto a tal punto da perdere "ciascun sentimento," cade "come l'uom cui sonno piglia." Lo spavento di Dante è simile al timore dei profeti dell 'Antico Testamento davanti alla manifestazione della divinità, al timore dei tre discepoli testimoni della trasfigurazione di Cristo e alla paura del centurione e degli altri che assistono alla morte di Cristo e ai segni straordinari che vi tengono dietro.

Dante sviene non solo perché la divinità manifesta la sua grandezza e terribilità, ma anche perché, davanti alla divinità, la creatura si rende conto della debolezza della propria natura, colpita dal peccato di Adamo e dal peccato personale. Ambedue gli aspetti sono presenti: prima, la debolezza della creatura dopo il peccato di Adamo, messa in evidenza dal verso "mi vinse ciascun sentimento"; poi, la peccaminosità del Pellegrino, sottolineata dalla caduta: "e caddi come l'uom cui sonno piglia." La sopraffazione dei sentimenti del Pellegrino è segno di quella profonda dicotomia effettuatasi in ogni discendente di Adamo come conseguenza diretta del peccato, per cui le forze superiori dell'uomo, l'anima e la mente, non solo non sono in grado di dominare le forze inferiori, cioè il corpo, ma esse stesse possono venir meno. La conseguenza più tragica di questa dicotomia inerente alla natura umana è appunto la morte. ${ }^{9}$ La perdita dei sensi di Dante, che ha la sua manifestazione esterna nella caduta, rivela la comunanza del Pellegrino con il resto dell'umanità negli effetti del peccato di Adamo e nella partecipazione personale al peccato.

La caduta "fisica" di Dante ha un antecedente mitico di fondamentale importanza teologica nella caduta dal cielo di Lucifero, come afferma Beatrice nell'ultimo sintagma del verbo "cadere" riscontrabile nella Commedia:

Principio del cader fu il maladetto superbir di colui che tu vedesti da tutti i pesi del mondo costretto.

(Par. 29.55-57)

Prefigurata in quella di Lucifero e di Adamo, la caduta di Dante sulla riva dell'Achcronte, il fiume della morte, è emblematica della debolezza della natura umana in genere e della peccaminosità del Pellegrino in particolare. 
Questa è dunque la condizione di Dante Pellegrino a questa congiuntura del viaggio. Arrivato all'Acheronte, il fiume della morte che separa questo mondo dall'aldilà, Dante si sente proclamare da Caronte che non gli è permesso di attraversare il fiume in quanto è "anima viva," cioè suscettibile di pentimento e quindi disponibile alla salvezza. A questo momento la divinità si manifesta nella sua terribilità, proclamando quindi di nuovo il suo dominio sopra il regno di Satana e mettendo in evidenza la debolezza del Pellegrino, soggetto al peccato di Adamo e responsabile della propria condizione peccaminosa. La condizione di Dante Pellegrino in seguito alla perdita della conoscenza, quindi, per alcuni aspetti è simile a quella di Cristo morto, per altri se ne discosta sostanzialmente. Cristo, pur essendo innocente, si assoggetta volontariamente alla morte, conseguenza del peccato; Dante Pellegrino, benché non muoia fisicamente, esperisce tuttavia temporaneamente la perdita delle sue facoltà fisiche e intellettuali a causa della propria condizione di peccatore. Cristo, dopo la morte, discende agli inferi, ne prende dominio e libera le anime dei giusti dal Limbo; dopo lo svenimento emblematico della morte, Dante può iniziare il viaggio infernale grazie alla discesa di Cristo e alla sua presa di possesso del regno di Satana.

La morte di Cristo implica nel contempo la sua sottomissione, breve e temporanea, al regno di Satana, sotto il cui dominio si trova l'uomo soggetto al peccato di Adamo e al peccato personale. Lo svenimento di Dante assolve una funzione analoga alla morte di Cristo. Se, da un lato, il Pellegrino può contare fin dall'inizio del viaggio sull'intervento divino, dall'altro non può non condividere, per quanto brevemente, la sconfitta esperita da Cristo stesso e sottomettersi quindi alle forze del male e della morte.

5. Fondamentale, per l'esatta comprensione dell'intero episodio acheronteo, è l'esplicitazione dei motivi poetici sottesi alla decisione di Dante auctor di passare sotto silenzio non solo il modo (ed è proprio il "modo" del passaggio ad interessare quasi esclusivamente i critici) ma l'azione stessa del transitus. In altri termini, la questione che ora intendiamo affrontare brevemente concerne i motivi teologici e poetici che hanno motivato Dante auctor, che narra tutti gli altri transitus del Pellegrino, compreso quello che il Pellegrino, benché immerso nel sonno, attua passivamente per opera di Lucia durante 
la prima notte purgatoriale, a lasciare nel silenzio narrativo questo primo e fondamentale passaggio. Lal glossa dei commentatori antichi (Terpening 127-39), salvo qualche raro spunto, non ha fatto altro che sottolineare le difficoltà interpretative di questo silenzio poetico; i critici contemporanci, nonostante le molteplici implicazioni estetiche di questo silenzio narrativo, hanno trascurato del tutto questa questione. $^{10}$

In realtà il motivo fondamentale del silenzio poetico dantesco che investe il passaggio acheronteo del Pellegrino va situato nel contesto biblico e teologico esposto sopra, contesto che occorre illuminare ulteriormente tramite una serie di riflessioni derivanti dai molteplici rapporti esistenti fra verbum e silentium.

La Bibbia, alla morte di Cristo, sottolineata incisivamente da un'ultima emissione di voce ("Iesus autem iterum clamans voce magna, emisit spiritum," Matt. 27.50) e alla sua sepoltura, passa sotto silenzio la condizione di Cristo da quel momento fino all'alba della domenica di resurrezione. Gli scrittori sacri infatti non descrivono che cosa avviene a Cristo durante quel periodo che nella liturgia viene chiamato triduo sacro. È proprio questo silenzio narrativo degli autori sacri ad indurre gli scrittori degli apocrifi ad amplificare il testo biblico descrivendo il descensus ad inferos.

Al silenzio narrativo biblico corrisponde il silenzio liturgico con cui la Chiesa commemora la morte di Cristo durante la liturgia del Venerdi e del Sabato Santo. La lettura del Passio, infatti, viene interrotta per commemorare in silenzio la morte di Cristo." Inoltre, nella tradizione giudeo-cristiana due elementi, apparentemente contraddittori ma in realtà integrantisi, coesistono all'interno della concezione della divinità. Dio è logos e sigé, verbum e silentium. Se è vero che nel tempo Egli rivela se stesso tramite la parola, tuttavia Egli continua a manifestarsi anche nel silenzio. La creatura, in pellegrinaggio verso la patria celeste, si accosta alla divinità tramite il verbum e il silenzio della stessa nei suoi rapporti con la creatura. La divinità, insomma, si configura non solo come deus revelatus ma anche come deus absconditus: ambedue gli aspetti condizionano il dialogo con la creatura. ${ }^{12}$ In breve, il silenzio poetico a riguardo del transiuss dell'Acheronte va situato in un contesto assai complesso, che deriva da varie tradizioni con cui il testo dantesco intreccia molteplici rapporti. ${ }^{13}$ Occorre dunque pensare alla divinità in quanto 
deus absconditus e silentium; al silenzio che accompagna la morte di Cristo nel narrato biblico e che caratterizza l'attuazione liturgica della morte di Cristo nella preghiera della Chiesa; al mistero che avvolge il passaggio da questa vita all'altra.

$\mathrm{Al}$ di là di questa reticolo di rapporti occorre sottolineare il modo in cui il verbum si adegua al factum poetico. Infatti è la perdita dei sensi del Pellegrino, cioè la realtà poetica che Dante vuole esprimere, a determinare la poesia, che così diventa "muta" o "morta" (si veda Purg. 1.7). Per quanto concerne il testo dantesco occorre sottolineare la condizione peculiare del Pellegrino durante il transitus. Poiché ha perso i sensi, non può sapere in qual modo abbia varcato l'Acheronte. Conseguentemente Dante auctor non descrive il passaggio del fiume proprio perché, non atteggiandosi ad autore onnisciente, non può sapere come esso sia avvenuto né può quindi descriverlo. Il silenzio narrativo che avvolge il passaggio dell'Acheronte deriva quindi dalla mancata comprensione dell'evento, incomprensione da parte dell'agens che l'auctor deve necessariamente rispettare, per motivi intrinsici ed estrinsici, quando narra l'evento post factum, cioè al termine del viaggio nell'aldilà. Questo silenzio poetico va quindi situato all'interno della concezione medievale del verbum, che San Tommaso esprime lucidamente:

Manifestius autem et communius in nobis dicitur verbum quod voce profertur. Quod quidem ab interiori procedit quantum ad duo quae in verbo exteriori inveniuntur, scilicet vox ipsa, et significatio vocis. Vox enim significat intellectus conceptum, secundum Philosophum, in libro I Periherm. [Bekker 16a3]: et iterum vox ex imaginatione procedit, ut in libro De anima [Bekker 420b32] dicitur. Vox autem quae non est significativa, verbum dici non potest. Ex hoc ergo dicitur verbum vox exterior, quia significat interiorem mentis conceptum. Sic igitur primo et principaliter interior mentis conceptus verbum dicitur: secundario vero, ipsa vox interioris conceptus significativa: tertio vero, ipsa imaginatio vocis verbum dicitur. Et hos tres modos verbi ponit Damascenus [De fide orth.: MG 94, 857], in I libro, cap. 13, dicens quod verbum dicitur naturalis intellectus motus, secundum quem movetur et intelligit et cogitat, velut lux et splendor, quantum ad primum: rursus verbum est quod non verbo profertur, sed in corde pronuntiatur, quantum ad tertium: rursus etiam verbum est angelus, idest nuntius, intelligentiae, quantum ad secundum.-Dicitur autem figurative quarto modo verbum, id quod verbo significatur vel efficitur: sicut consuevimus dicere, hoc est verbum quod dixi tibi.... (STh. 1 q. 34 a. 1 , Respondeo $)^{14}$ 
Nel contesto, quindi, di queste riflessioni tomistiche circa il ierbum, è possibile delucidare ulteriormente il significato dello svenimento del Pellegrino e il silenzio poetico circa il passaggio acheronteo. Il Pellegrino sviene perché è incapace di comprendere questo momento essenziale del passaggio da questo mondo all'aldilà: ${ }^{15}$ non solo il modo ma anche, e soprattutto, il significato del viaggio che sta per intraprendere. Non può comprendere perché è immerso nel peccato, cioè "sonno" o privazione dei sensi, che caratterizza, rispettivamente, il suo ingresso nella selva oscura $e$ il passaggio del fiume. Appunto perché è immerso nel peccato, sonno interiore o privazione dei sensi, non può comprendere ciò che la divinità sta attuando in lui tramite il viaggio nell'aldilà. All'incomprensione dell'intelletto segue necessariamente il silenzio circa l'evento stesso.

Come al conceptus intellectus corrisponde prima il verbum mentale e poi il verbum o vox exterior, alla mancata comprensione di un evento da parte dell'intelletto non può corrispondere che il silentium mentale e quindi il silenzio narrativo o poetico quale unica adeguata corrispondenza fra realtà e forma espressiva. Incomprensione e silenzio, quindi, essenzialmente connessi con lo smarrimento e sonno descritti all'inizio del viaggio nel primo canto dell'Inferno e con lo svenimento e la caduta di Dante Pellegrino lungo la riva dell'Acheronte. Questa negatività-morale, intellettiva, verbale-si trasforma nell'assenza del narrato. Questo silenzio narrativo, tuttavia, assurge immediatamente a funzione poetica estremamente efficace: non solo perché esso è l'unico mezzo disponibile all'auctor ma anche, e soprattutto, perché questa "morte" della poesia esprime in forma poetica perfettamente adeguata l'incomprensione dell'evento da parte dell'agens e la sua morte spirituale. ${ }^{16}$

\section{University of North Carolina at Chapel Hill}

\section{NOTE}

1 Charles S. Singleton, nel saggio "Le visuali retrospettive," sviluppa una valida analisi delle tre "ruine," partendo dalla terza, quella annunciata da Malacoda, e ponendole appunto nel contesto della morte di Cristo e del terremoto che ne consegue: "Tutte e tre sono prove del fendersi delle rocce in quel gran momento del calendario cristiano in cui il Redentore morì sulla Croce. É così che I'Inferno attesta quella Morte che è la nostra salvezza dalla seconda morte: le 
tre ruine marcano quel momento, imprimendone nella roccia dell'Inferno tre eterni segnali" (La poesia 481). Singleton conclude la sua analisi sviluppando un rapporto stretto fra le tre "ruine" quali eterni segnali impressi nella roccia dell'inferno non solo, come è ovvio, con la morte di Cristo ma anche con l'inizio del viaggio dantesco. La "discesa," infatti, inizia verso l'imbrunire (si veda Inf. 2.1-2), Dante e Virgilio raggiungono il centro della terra circa le sei di sera del Sabato Santo (Singleton 487), iniziano la scalata verso il Monte Purgatorio verso le 7,30 del Sabato Santo, a causa dell'anticipazione di dodici ore (Singleton 487), e raggiungono le sponde della montagna sacra la mattina della Domenica di Pasqua.

2 R. G. Austin definisce il primo elemento "A conventional detail" (Aeneidos 113 ; n. al v. 256) e rimanda a $E n, 4.490-91$ ("mugire videbis / sub pedibus terram et descendere montibus ornos") e a Ovidio, Metam. 14.409-10 ("lapides visi mugitus edere raucos / et latrare canes"). Austin fa altri riferimenti per il latrare dei cani nella sua nota.

3 Ulteriori differenze risultano dall'analisi dei sintagmi connotativi, nel testo virgiliano e dantesco, della condizione di Enea, Dante e delle anime. Nell'Eneide abbiamo in riferimento ad Enea e ai morti: "corpora viva nefas Stygia vectare carina" (6.391); "ingentem Aenea" (6.413); "et ferruginea [Charon] subvectat corpora cumba" (6.303); "matres atque viri defunctaque corpora vita magnanimum heroum" (6.306-7); "animae" (6.319). Nel testo dantesco i sintagmi seguenti sono connotativi della condizione privilegiata del Pellegrino, chiamato da Caronte "anima viva" (3:88) e, indirettamente e implicitamente, da Virgilio "anima buona" (3.127); le anime dei dannati, al contrario, sono chiamate "anime prave" (3.84), "morti" (3.89), "anime . . lasse e nude" (3.100), "il mal seme d'Adamo" (3.115), "quelli che muoion ne l'ira di Dio" (3.122).

4 Un'analisi del terremoto in Inferno 3 e Purgatorio 20-21 mette chiaramente in evidenza che il primo è annunciatore di morte mentre il secondo è annunciatore di vita: ambedue derivanti il loro valore dall'evento cristologico.

5 La Sainte bible: Mt 27.51 nota o; Mt 24.1 nota d.

6 Il narrato virgiliano della visita di Beatrice nel Limbo appartiene, come è ovvio, all'antefatto della storia ed è situato, spazialmente e temporalmente, nel Limbo, cioè nell'aldilà.

7 Questa concezione, l'Acheronte quale divisione fra evo presente e l'aldilà, è proposta, per quanto implicitamente, da Charles S. Singleton: "In un certo senso, si potrebbe desiderare che alla fine del secondo canto dell'Inferno calasse un sipario, per distinguere da ciò che segue il prologo del poema costituito dai primi due canti" (La poesia 26).

8 “. . f factae sunt tenebrae . . . in signum futurarum tenebrarum, quae comprehensurae erant gentem Judaeam. . . . sub Christo autem factae sunt tenebrae super omnem terram Judaeam tribus horis: quoniam propter peccata sua privati sunt a lumine Dei Patris, et a splendore Christi, et ab illuminatione Spiritus sancti" (S. Tommaso, Catena aurea 1: 451; Matt. 27).

9 “. . naturali morte moriuntur omnes communiter, tam nocentes quam inno- 
centes. Quae quidem naturalis mors divina potestate inducitur propter peccatum originale; secundum illud I Reg. 2.6: Dominus mortificat ct vivificat" (STh la 2ac, q. 94, a. 5 ad 2).

10 Secondo R. Hollander, Dante auctor avrebbe deciso di passare sotto silenzio il passaggio acheronteo perché la narrazione sarebbe risultata "too self-consciously redolent" del passaggio acheronteo di Enea (292 n. 5).

11 "Et inclinato capite tradidit spiritum. (Hic genu flectitur, et pausatur aliquanuulum.)" (Missale Romanum 153). Nella liturgia del venerdi santo si veda anche: "Finito Tractu, dicitur Passio super nudum pulpitum: quam Celebrans submissa voce legit in cornu Epistolac" (Missale Romanum 150). "Non dicitur 'Corpus tuum, Domine', nec Postcommunio, nec 'Placeat tibi', nec datur benedictio: sed facta reverentia Altari, Sacerdos cum Ministris discedit: ct dicuntur Vesperae sine cantu, et denudatur Altare" (Missale Romanum 160). Parimenti viene anche sospeso il suono delle campane.

12 Per una discussione del silenzio nella Bibbia si veda la voce "Silenzio" nel Vocabulaire de théologie biblique, dove si nota che nella Bibbia il silenzio c̀ indice della collera divina (Ez. 3.26), segno di punizione (Is. 64.11), allontanamento del Signore (Ps. 35.22), "un arrêt de mort" (Ps. 28.1), c annunzia lo Sheol, dove Dio e l'uomo non si parlano più (Ps. 94.17; 115.17). Per una trattazione più ampia e generale rimando al volume di Gustav Mensching. In chiave moderna, e nel contesto di una trattazione generale della retorica come "filosofia postfilosofica," fondamentali sono le pagine di Paolo Valesio, soprattutto 353-97.

13 Occorre anche accennare ad un contesto che è basato su motivi psicologici. Se è vero, come si è proposto sopra, che la caduta-svenimento di Dante c̀ da interpretarsi anche come una forma di morte fisica, allora è possibile proporre che il silenzio poetico sottolinei il mistero che avvolge la morte di ogni uomo: mentre la vita terrena, sperimentata e sperimentabilc, può essere descritta tramite i mezzi verbali disponibili c l'esperienza dell'aldilà presenta problemi di comunicazione variamente risolvibili, il passaggio dalla prima alla seconda potrebbe non scmbrare riducibile ai mezzi espressivi che pertengono a queste due esperienze.

$14 \mathrm{Si}$ veda anche: "Si autem dicitur verbum quia exterius manifestat, ea quae exterius manifestant, non dicuntur verba nisi inquantum significant interiorem mentis conceptum, quem aliquis etiam per exteriora signa manifestat" (STh. 1 q. 34 a. 1, Ad primum). "Cum ergo dicitur quod verbum est notitia, non accipitur notitia pro actu intellectus cognoscentis, vel pro aliquo eius habitu: sed pro eo quod intellectus concipit cognoscendo. Unde et Augustinus dicit quod Verbum est sapientia genita: quod nihil aliud est quod ipsa conceptio sapientis: quae ipsa pari modo notitia genita dici potest" (STh. 1 q. 34 a. 1 , Ad secundum). "Nam intelligere importat solam habitudinem intelligentis ad rem intellectam. ... Sed dicere importat principaliter habitudinem ad verbum conceptum: nihil enim est aliud dicere quam proferre verhum. Sed mediante verbum importat habitudinem ad rem intellectam, quae in verbo prolato 
manifestatur intelligenti" (STh. 1 q. 34. a 1, Ad tertium).

$15 \mathrm{Su}$ questa incomprensione si veda quanto ho scritto in Dante's Poetry of Dreams cap. 5 , in riferimento anche agli altri sonni e perdite di coscienza di Dante.

16 Come lo svenimento del Pellegrino è stato posto in relazione con il "sonno," causa dello smarrimento nella selva oscura, anche il silenzio narrativo che avvolge il passaggio dell'Acheronte è annunciato dalla incapacità o difficoltà di Dante auctor a descrivere la foresta ("Ahi quanto a dir qual era è cosa dura / esta selva selvaggia e aspra e forte," Inf. 1.4-5) o a spiegare come Dante vi sia entrato ("Io non so ben ridir com'i' v'entrai," Inf. 1.10). In altra sede intendo ritornare più ampiamente sul valore di questo silenzio poetico nel contesto di tutta la Commedia e nei suoi molteplici rapporti con la parola umana e il verbo divino.

\section{OPERE CITATE}

Alighieri, Dante. La Commedia secondo l'antica vulgata. A cura di Giorgio Petrocchi. 4 voll. Milano: Mondadori, 1966-67.

. Tutte le opere. A cura di Fredi Chiappelli. Milano: Mursia, 1965.

Biagi, G. et al. (A cura di). La Divina Commedia nella figurazione antica e nel secolare commento. 3 voll. Torino: UTET, 1931.

Biblia sacra iuxta vulgatam clementinam. Madrid: BAC, 1982.

Cervigni, Dino S. Dante's Poetry of Dreams. Biblioteca dell" Archivum Romanicum" 198. Firenze: Oischki, 1986.

Hollander, Robert. "Dante on Horseback (Inferno XII, 93-126)." Italica 61 (1984): 287-96.

Iannucci, Amilcare. Forma ed evento nella Divina Commedia. Strumenti di ricerca 41-42. Roma: Bulzoni, 1984.

Mensching, Gustav. Das Heilige Schweigen. Eine Religionsgeschichtliche Untersuchung. Giessen: A. Töpelmann, 1926.

Missale romanum ex decreto sacrosancti concilii tridentini restitutum, sancti Pii V. Pontificis Maximi jussu editum . . . Baltimori, 1835.

La Sainte bible. Traduite en français sous la direction de l'École Biblique de Jérusalem. Paris: Éditions du Cerf, 1956.

Servio. Servii grammatici qui feruntur in Vergilii Carmina commentarii. Vol. 1. Aeneidos librorum I-V commentarii. Recensuit Georgius Thilo. Lipsiae, 1881.

Singleton, Charles S. La poesia della Divina Commedia. Bologna: Il Mulino, 1978. Trad. di Commedia. Elements of Structure. Cambridge: Harvard UP, 1957; Journey to Beatrice. Cambridge: Harvard UP, 1958.

Terpening, Ronnie H. Charon and the Crossing: Ancient, Medieval and Renaissance Transformations of a Myth. Lewisburg: Bucknell UP, 1985.

S. Tommaso D'Aquino. Summa Theologiae. 5 voll. Madrid: BAC, 1965-68. . Catena aurea in quatuor evangelia. 2 voll. Torino: Marietti, 1888-89.

Valesio, Paolo. Ascoltare il silenzio: la retorica come teoria. Bologna: Il Mulino, 1986. 
Virgilio. Aeneidos. Liber sextus. Commentary by R. G. Austin. Oxford: Clarendon Press, 1977.

Vocabulaire de théologie biblique. Publić sous la direction de X. Léon-Dufour et a). Paris: Les Éditions du Cerf, 1964. 